\title{
WDM-PON Based on Wavelength Locked Fabry-Perot LDs
}

\author{
Chang-Hee Lee* and Sil-Gu Mun \\ Dept. of EECS, KAIST, 373-1 Guseong-dong, Yuseong-gu, Daejeon, 305-701, South Korea
}

(Received December 10, 2008 : accepted December 12, 2008)

\begin{abstract}
A WDM-PON has been considered as an ultimate solution for access networks. However, there were many technical and practical issues for commercial deployment. These issues were solved with wavelength locked F-P LD and the WDM-PONs employing this optical source were commercialized. These WDM-PON systems have been deployed in Korea, Europe, and US. We reviewed wavelength locking technology and WDM-PON achievements.

When we inject spectrum sliced broadband light into an F-P LD, the multimode output is changed to a quasi single mode. Then, we can use the single mode light for WDM signal transmission. The broad spectral gain of the semiconductor gain medium enables a color-free operation of WDM-PON, i.e., an identical ONT can be used for each user. The wavelength locking properties depend on many parameters, especially alignment of injection wavelength to a lasing mode, passband profile of AWG and front facet reflectivity of F-P LD. However, these dependencies can be reduced by proper design of the laser and the injection bandwidth. Thus, WDM-PON systems have been achieved with color-free operation.
\end{abstract}

Keywords: WDM-PON, Access network, FTTH, Wavelength-locking

OCIS codes : (060.0060) Fiber optics and optical communications; (060.4230) Multiplexing;

(060.4250) Networks; (060.4510) Optical communications

\section{INTRODUCTION}

Recently, voice and text oriented services have been evolved to data and image based services due to the popularity and growth of the internet. This evolution is continuing towards video based services that will require much higher data speeds. In addition, it has been observed that the traffic patterns are becoming more and more symmetric [1]. This change of paradigm will require new access networks that support high-speed $(>100 \mathrm{Mb} / \mathrm{s})$, symmetric, and guaranteed bandwidths for future video services with high-definition TV (HDTV) quality. Thus the access network should be prepared and structured to cover voice, data and video traffic with a focus on higher bandwidth applications such as video and image services. All traffic in the access network began to merge to Internet Protocol (IP) packet and IP video will be a common platform for video services.

When all services become video-centered, it is not expected that statistical multiplexing gain, as in the

*Corresponding author: chl@ee.kaist.ac.kr data-centric networks, will be that useful. Future access networks will need to offer guaranteed-symmetric bandwidths of $100 \mathrm{Mb} / \mathrm{s}$ [2] instead of only a peak bandwidth of $100 \mathrm{Mb} / \mathrm{s}$ that is shared with many subscribers. In addition, the transmission lengths should be designed to meet $20 \mathrm{~km}$, which is the established international standard based on distances from subscribers to central office (CO) locations. Furthermore, long reach transmission has been considerable attention for skipping of COs. This will reduce service provider's operational expense (OPEX). It also will reduce the power consumption in communication networks by reducing network elements.

It is well known that a single-mode fiber (SMF) is the transmission medium in future access networks, since it is the only medium that supports high capacity traffic $(>1 \mathrm{~Gb} / \mathrm{s})$ over $20 \mathrm{~km}$. The ultimate goal in access network is to provide an optical fiber to each customer premise or home. Fig. 1 shows four different architectures in access networks based on optical fiber. Among them, a passive optical network (PON) that uses a point-tomultipoint architecture as shown in Fig. 1 (c) and (d) 

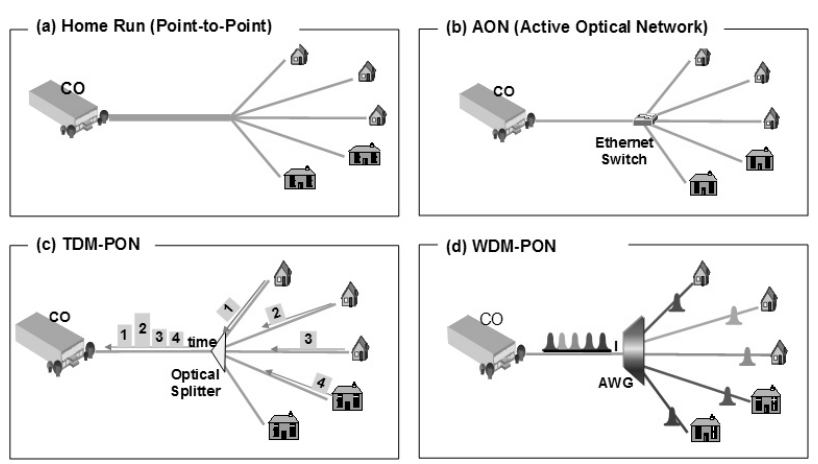

FIG. 1. Four different approaches of FTTH/P, (a) pointto-point connection, (b) active optical network that has active equipments in the field, (c) and (d) passive optical network.

has been commonly used due to the passive nature of the architecture. The term PON means that there are no active elements between the $\mathrm{CO}$ and the customer premises. In other words, only optical fibers and optical passive elements that do not require any electrical power or active management are used. It should be noted that the lifetime of outside plant (OSP) is greater than 25 years, since it is realized with only passive devices. This feature justifies installation costs and minimizes the OPEX. The PON with optical power splitter as an optical branching device uses many different multiple access methods to share a single strand of fiber from the CO to remote node (RN) [3]. Here, we define RN as optical branching device for PON. The most common method is the time division method, called as time division multiplexing-PON (TDM-PON). As a different approach, the RN can be realized with a wavelength division multiplexing/demultiplexing device to accommodate wavelength division multiplexing-PON (WDMPON).

A TDM-PON is starting to see significant deployments in various regions of the world. In this architecture, 'N' users share a single upstream and downstream transmission channel. Thus the transmission data rate over the PON must be $\mathrm{N}$ times the dedicated data rate to each subscriber. This approach can provide a costeffective solution since only a single transmitter and receiver is shared at the $\mathrm{CO}$ location. Currently, E-PON and G-PON are deploying in the field. The E$\mathrm{PON}$ that carries $1.25 \mathrm{~Gb} / \mathrm{s}$ data rate for both upstream and downstream has been used in Asia, while G-PON that carries $2.5 \mathrm{~Gb} / \mathrm{s}$ data rate and $1.25 \mathrm{~Gb} / \mathrm{s}$ data rate for downstream and upstream, respectively, has been used in other regions of the world. These solutions are suitable as long as the bandwidth demand of the many users sharing the PON does not become too great. To address these limited bandwidth concerns, some TDMPON have added a separate video overlay wavelength to provide TV signals that are compatible with existing
TV sets and home wiring. However, this is not suitable for IPTV services which require higher dedicated bandwidth. The TDM-PONs which has higher bandwidth are actively discussed in two standard bodies of Institute of Electrical and Electronics Engineers (IEEE) and Full Service Access Network (FSAN) to meet future bandwidth requirements. This problem is more challenging than in other fast changing technology markets since these choices affect wide spread infrastructure decisions which hopefully should last for 25 years or more.

A WDM-PON provides an optical point-to-point connection by allocating a pair of wavelengths to each user connected to the PON and solves many problems originated by bandwidth sharing. The only difference in the OSP is replacing the optical power splitter in a TDM-PON with an arrayed waveguide grating (AWG) to demultiplex the downstream wavelengths and multiplex the upstream wavelengths. The wavelength channel spacing in an AWG can be $0.8 \mathrm{~nm}$ or less which allows many users to be connected to a WDM-PON. In addition to its efficient use of wavelengths, a WDM-PON also has advantages in its use of optical transmission power. TDM-PON architectures typically use a $1 \times 32$ power splitter at the RN that results in an insertion loss of about 17 or $18 \mathrm{~dB}$. In contrast, the loss through typical AWGs can be in the range of 3 to $5 \mathrm{~dB}$. Although 2 AWGs are required in WDM-PON, the insertion loss improvement between a transceiver pair is on the order of 8 to $11 \mathrm{~dB}$. In addition to the much lower insertion loss, an additional optical efficiency occurs since the noise bandwidth of each receiver can be decreased to $1 / \mathrm{N}$ compared with a TDM-PON approach [3]. For a PON with 32 users, this can result in an additive improvement of about $10 \mathrm{~dB}$ in the receiver sensitivity. This improvement becomes even larger when the PON is scaled up to service more users since the insertion loss through an AWG is effectively independent of its splitting ratio. Compared with a TDM-PON, the WDMPON approach offers large improvements in both optical power penalty and its ability to scale up to serve a large number of users sharing a single PON. These features are also important for realizing a long reach PON [4].

These efficiency improvements are very important to the upgradeability and flexibility of a WDM-PON system. Since each user has a point-to-point wavelength connection, it is possible to provide different data rates and protocols to each subscriber. Since each user is uncoupled from others on the PON, data rate upgrades can be done on an individual basis without causing disruption to any of the other users. Using an AWG splitter with a $100 \mathrm{GHz}$ channel spacing, it would be possible to provide future bandwidths of up to about $40 \mathrm{~Gb} / \mathrm{s}$ to each user. This flexible bandwidth upgradeability would be very useful if a WDM-PON is shared with a diverse set of users, such as business customers and home resi- 
dents. There would be no issues regarding quality of service (QoS) or privacy since no optical signals are shared with anyone else connected to the PON. An additional feature of the WDM architecture is its ability to localize any fault or optical loss in the fiber plant by using a single wavelength-tunable OTDR located at the $\mathrm{CO}[5]$. This could yield large operational and maintenance savings compared to PONs that use power splitters since it is very difficult to isolate problems in the fiber plant past the position of the RN.

In this paper, we review the WDM-PON based on wavelength locked F-P LD. In section II, we describe main principles and technologies of the wavelength locking. Then, we describe research and development activities in section III. The summary is presented in section IV.

\section{WDM-PON BASED WAVELENGTH LOCKED F-P LD}

\section{A. Wavelength locked F-P LD}

Although a WDM-PON has many technical advantages, there have been several issues that have prevented it from being a suitable solution for access applications. One issue has been related to the wavelength stability of the AWG located at the passive RN. Until recently, AWG required temperature control to keep their optical channels locked to a wavelength grid. This would have required electric power at the $\mathrm{RN}$ that would be unacceptable for a PON solution. Technology advances have allowed the recent commercialization of athermal AWGs that can remain locked to a DWDM wavelength grid over temperature ranges experienced at the passive node location [6]. It is also projected that these athermal AWGs will have costs similar to optical power splitters since each will use the same planar lightwave circuit (PLC) technology, and the major costs are in the packaging which will be similar for each device.

Another issue is the concern of using wavelengthspecific sources such as distributed feedback (DFB) lasers in a WDM-PON system. If DFB lasers were used, these lasers would require thermo-electric coolers (TEC) to stabilize their wavelengths that would result in relatively expensive packaging. Also, this scheme would require a different or 'colored' transceiver for each user, resulting in high costs for inventory management and maintenance. To address these issues, much effort has been done on trying to develop low-cost sources that are 'color-free' or wavelength independent so that all subscribers can have identical optical network terminations (ONTs). Some of these approaches are listed in Table 1. It may also be possible to provide color-free operation by using relatively expensive wavelength tunable lasers if prior information of the channel wavelength is known [7].

One promising approach has recently been proposed that enables color-free and identical transmitters by using an automatic wavelength-locking scheme [8]-[10]. This scheme relies on providing an optical seeding signal generated at the optical line termination (OLT) that can be used to wavelength-lock the identical optical transmitters located at each remote ONT. The

TABLE 1. Various optical sources for WDM-PON

\begin{tabular}{|c|c|c|c|c|c|c|c|c|}
\hline & & & $\begin{array}{l}\text { Wavelength } \\
\text { control }\end{array}$ & $\begin{array}{l}\text { Modulation } \\
\text { Scheme }\end{array}$ & $\begin{array}{l}\text { Modulation } \\
\text { speed }\end{array}$ & $\begin{array}{l}\text { Color- } \\
\text { free ONT }\end{array}$ & $\begin{array}{l}\text { Operation } \\
\text { bandwidth }\end{array}$ & Remarks \\
\hline \multirow{5}{*}{$\begin{array}{l}\text { Solitary } \\
\text { source } \\
\text { at ONT }\end{array}$} & \multicolumn{2}{|c|}{ Wavelength specific laser } & Needed at ONT & Direct & $10 \mathrm{~Gb} / \mathrm{s}$ & No & Unlimited & \\
\hline & \multicolumn{2}{|c|}{ Tunable laser } & Needed at ONT & Direct & $\sim 2.5 \mathrm{~Gb} / \mathrm{s}$ & Yes & $40 \mathrm{~nm}$ & $\begin{array}{l}\text { - Wavelength } \\
\text { information }\end{array}$ \\
\hline & \multirow{3}{*}{$\begin{array}{l}\text { Broadband } \\
\text { light: ASE } \\
\text { from }\end{array}$} & LED & No & Direct & $\sim 100 \mathrm{Mb} / \mathrm{s}$ & Yes & $>50 \mathrm{~nm}$ & \multirow{3}{*}{$\begin{array}{l}\text { - High slicing loss. } \\
\text { - Dispersion limit for } \\
>1 \mathrm{~Gb} / \mathrm{s}\end{array}$} \\
\hline & & SLD & No & Direct & $<1 \mathrm{~Gb} / \mathrm{s}$ & Yes & $>50 \mathrm{~nm}$ & \\
\hline & & $\begin{array}{l}\text { Doped } \\
\text { fiber }\end{array}$ & No & External & $>10 \mathrm{~Gb} / \mathrm{s}$ & Yes & $30 \mathrm{~nm}$ & \\
\hline \multirow{7}{*}{$\begin{array}{l}\text { Seed } \\
\text { from } \\
\text { OLT }\end{array}$} & \multirow{3}{*}{$\begin{array}{l}\text { Array of } \\
\text { wavelength } \\
\text { specific } \\
\text { laser at } \\
\text { OLT }\end{array}$} & $\begin{array}{l}\text { External } \\
\text { modulator }\end{array}$ & Needed at OLT & External & $2.5 \mathrm{~Gb} / \mathrm{s}$ & Yes & Unlimited & \multirow{3}{*}{$\begin{array}{l}\text { - Back reflection penalty } \\
\text {-Two feeder fibers } \\
\text { - Needs optical amplifier } \\
\text { for EM }\end{array}$} \\
\hline & & RSOA & Needed at OLT & Direct & $\sim 1 \mathrm{~Gb} / \mathrm{s}$ & Yes & $>50 \mathrm{~nm}$ & \\
\hline & & F-P LD & $\begin{array}{l}\text { Needed at OLT } \\
\text { and ONT }\end{array}$ & Direct & $\sim 2.5 \mathrm{~Gb} / \mathrm{s}$ & Yes & $50 \mathrm{~nm}$ & \\
\hline & \multirow{3}{*}{$\begin{array}{l}\text { Broadband } \\
\text { light at OLT }\end{array}$} & $\begin{array}{l}\text { External } \\
\text { modulator }\end{array}$ & No & External & $>10 \mathrm{~Gb} / \mathrm{s}$ & Yes & \multirow{3}{*}{$\begin{array}{l}30 \sim 50 \mathrm{~nm} \\
\text { (Limited by } \\
\text { broadband } \\
\text { light at OLT) }\end{array}$} & \multirow{3}{*}{$\begin{array}{l}\text { - High power seed light } \\
\text { - Dispersion limit for } \\
>1 \mathrm{~Gb} / \mathrm{s} \\
\text { - Needs optical amplifier } \\
\text { for EM }\end{array}$} \\
\hline & & RSOA & No & Direct & $\sim 1 \mathrm{~Gb} / \mathrm{s}$ & Yes & & \\
\hline & & F-P LD & No & Direct & $\sim 2.5 \mathrm{~Gb} / \mathrm{s}$ & Yes & & \\
\hline & \multicolumn{2}{|c|}{$\begin{array}{l}\text { Remodulation of } \\
\text { downstream data }\end{array}$} & Needed at OLT & $\begin{array}{l}\text { Direct/ } \\
\text { External }\end{array}$ & $\sim 2.5 \mathrm{~Gb} / \mathrm{s}$ & Yes & $50 \mathrm{~nm}$ & $\begin{array}{l}\text { - Back reflection penalty } \\
\text { - Limited dynamic range }\end{array}$ \\
\hline
\end{tabular}




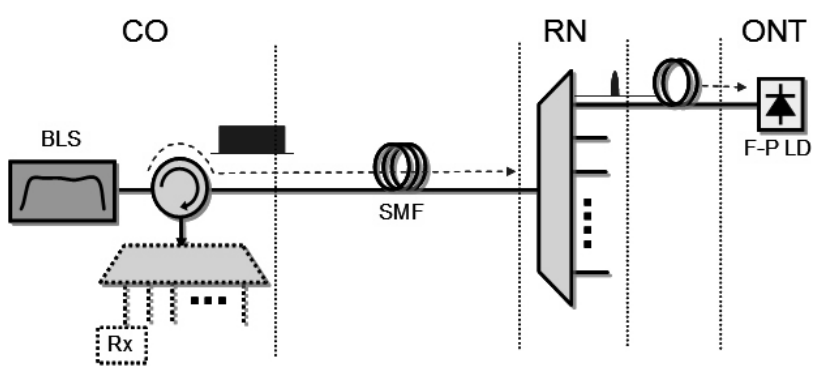

(a)
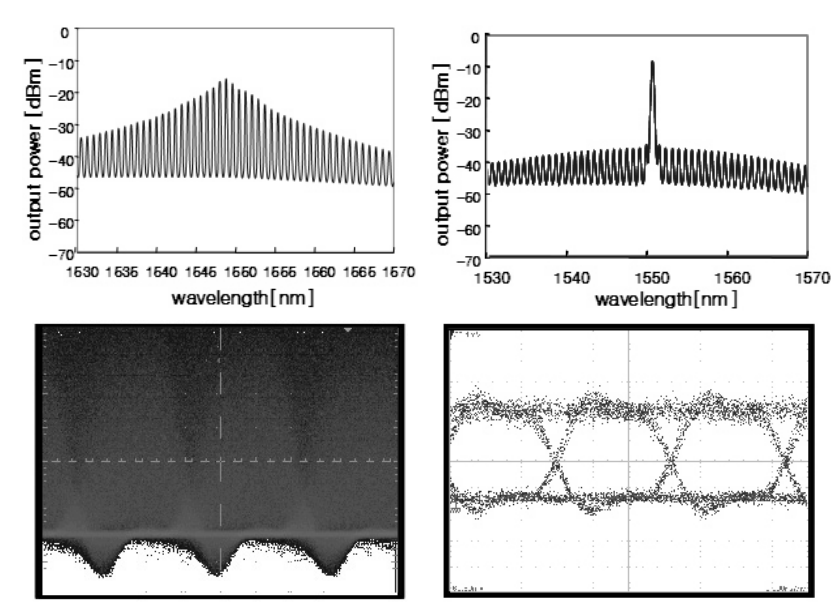

(b)

(c)

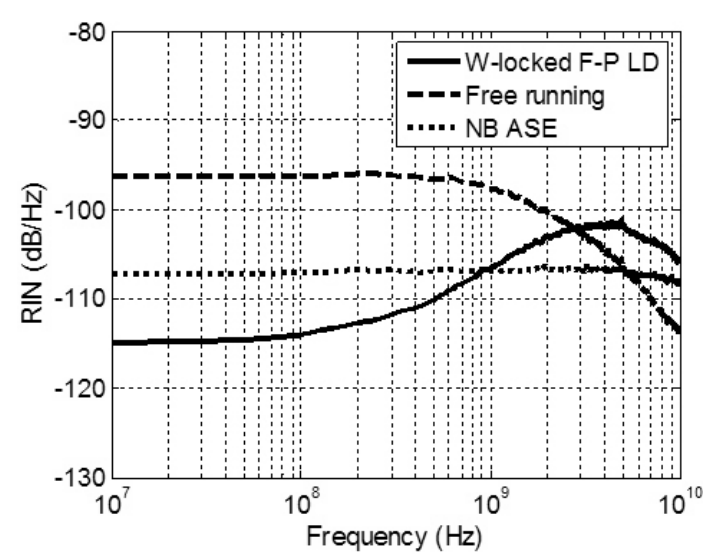

(d)

FIG. 2. (a) Schematic diagram of wavelength-locked F-P LD, (b) the output spectrum and eye-diagram before wavelength-locking, (c) those after wavelength-locking, and (d) the measured RIN with (thick line) and without (dashed line). The dotted line represents the RIN of the injected ASE.

identical ONT lasers can be simple Fabry-Perot laser diodes (F-P LD) [8] or reflective optical semiconductor amplifiers (RSOA) [9]. The F-P LD or RSOA performs three functions; amplification, data modulation and reflection of the reference seed signal provided by the OLT. The reference seed signal can be generated by an array of wavelength-specific sources such as DFB lasers or by a simple broadband amplified spontaneous emission (ASE) light source. Since this reference signal has to pass through the wavelength passband of the AWG at the RN, each of the identical ONTs is automatically aligned to the optical channel defined by the WDM-PON infrastructure. Recently, commercial WDM-PON systems based on wavelength locked F-P LD have been deployed in Korea [10]. It may also be possible to use the downstream data signal as a seed for the ONT transmitter, but large penalties result in both dynamic range and ONT complexity [11].

It is well known that an F-P laser shows multimode oscillation. The F-P laser output shows a huge amount of mode partition noise (MPN) when only a single mode is selected among the multimode output. This is an intrinsic feature in a multimode laser with a homogeneously broadened gain medium. Competition among lasing modes caused by fluctuation of spontaneous emission prevents steady oscillation of each mode in the time domain. The semiconductor F-P LD shows multimode oscillation as a common multimode laser, since the spontaneous emission is almost wavelength independent and the gain spectrum is very broad compared with the mode spacing. Thus, it is impossible to use a selected single mode light from the F-P LD for optical communications. Fortunately, it is possible to generate a single longitudinal mode light by injecting a narrow band spontaneous emission into the F-P LD. In this case, injected spontaneous emission is amplified selectively when the injected light power is much higher than the spontaneous emission generated by the gain medium. The experimental set-up to demonstrate this principle is shown in Fig. 2. It should be noted that the F-P LD may be the most efficient light source for optical communications while also being cost effective.

This setup represents the upstream data transmission configuration in a real system. The broadband light source (BLS) and the receiver including a wavelength division demultiplexer are located at a $\mathrm{CO}$, while the F-P LD is located at the customer's premises. The AWG is located at a RN. The BLS by a pumped erbium-doped fiber (EDF) generates ASE light. The bandwidth of the injected ASE is $0.4 \mathrm{~nm}$ which is equal to the $3 \mathrm{~dB}$ bandwidth of the AWG. We also used an anti-reflection (AR) coated F-P LD (as a cleaved back facet and $1 \%$ front facet reflectivity) with a cavity length of $600 \mu \mathrm{m}$. The injected power into the F-P LD (biased at 1.2 times the lasing threshold) is $-16 \mathrm{dBm} / 0.2 \mathrm{~nm}$. As shown in the measured spectrum, the output of the F-P LD becomes a single mode light after injection of a narrow band ASE. The linewidth of the F-P LD output is about $0.2 \mathrm{~nm}$. To see the suppression of the MPN, we also show the measured relative intensity noise (RIN) before and after injection. The low frequency RIN (solid line) mainly caused by mode-partition noise was suppressed by about $20 \mathrm{~dB}$ with injection compared with a single 


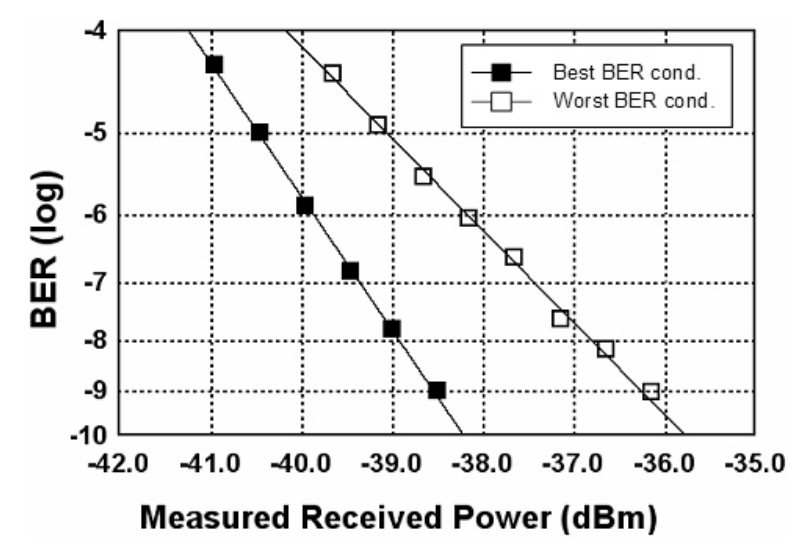

FIG. 3. The best and worst BER of wavelength-locked F-P LD.

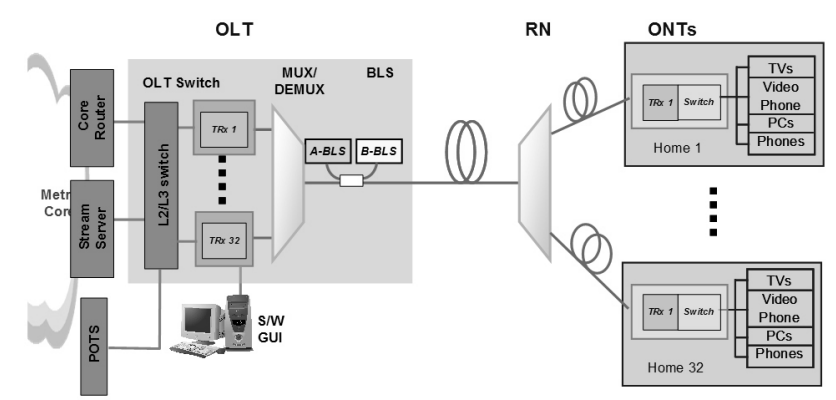

FIG. 4. Configuration of WDM-PON based on wavelength locked F-P LDs.

mode light of the free running laser (dashed line), as shown in Fig. 2 (d). The RIN of the output light is about $7 \mathrm{~dB}$ less than that of the injected narrow band ASE (dotted line). The side mode suppression ratio depends on the injection power, bias current, and detuning between the lasing mode and injection wavelength [12].

To investigate the possibility of data transmission with the wavelength locked F-P LD, we modulated the laser directly with a $155 \mathrm{Mb} / \mathrm{s}$ pseudo-random bit sequence (PRBS) of length $2^{23}-1$. The modulated signal was received by an optical receiver after transmission of a $20 \mathrm{~km}$ SMF. The measured eye-diagrams in Fig. 2 (b), (c) with and without injection show the effect of injection clearly. The completely closed eye without injection was opened with injection of the ASE. The measured BER curve also showed error-free transmission. It is predicted that the locking effect/efficiency will be the worst when the injection wavelength is located at the center of the adjacent modes. However, we can achieve error-free transmission with a $2.3 \mathrm{~dB}$ penalty, as shown in Fig. 3. It is noted that there was a negligible dispersion penalty. The gain bandwidth of the semiconductor active medium is typically broader than $50 \mathrm{~nm}$. Thus, it is possible to use a single type of F-P LD over an approximately equal wavelength range.
It should be noted that the backscattering of the injected ASE due to Rayleigh scattering and/or reflection at splice or connection points will interfere with the upstream signal. This interference induced penalty is negligibly small, since the band of the upstream signal (always broader than $0.1 \mathrm{~nm}$ ) is very wide compared with the receiver bandwidth. The measured penalty was less than $0.1 \mathrm{~dB}$ with a $30 \mathrm{~dB}$ reflection coefficient.

For downstream stream data transmission, another BLS and F-P LDs are located at the $\mathrm{CO}$ as shown in Fig. 4. The A-band wavelength and the B-band wavelength are separated by multiples of the AWG's free spectral range (FSR). In this manner, we consolidated multiplexing and demultiplexing in a single AWG. The athermal AWG whose transmission wavelengths have a very small temperature dependency [6] was used to eliminate the wavelength drift induced by temperature change.

A diplexer is needed for a transceiver both at the OLT and the ONT, since only a single strand of fiber is used for communication. Note that a TDM-PON also uses an optical diplexer. To make the diplexer cost effectively, a sufficient amount of guard band between the upstream and the downstream wavelength bands is needed. A conventional C-band $(1530-1560 \mathrm{~nm})$ was selected for the upstream transmission, since the loss of the fiber is minimal at this band. We chose E-band (1425 - 1450 $\mathrm{nm}$ ) for the upstream to have a sufficient guard band.

The performance of the WDM-PON can be dependent on many parameters. The most critical element in the WDM-PON with wavelength locked F-P LD is the laser itself. When we use uncoated laser (as cleaved facet), there exists a strong gain difference between the resonant case (injection wavelength matches to one of the lasing wavelength) and the non-resonant case (injection wavelength located in the middle of the two adjacent lasing mode). Then, both gain and side mode suppression ratio (SMSR) have maximum values for the resonant case, while these have minimum values for the non-resonant case. Consequently, the effects of injection diminish as the injection wavelength departs from the lasing mode. The worst case appears when the injection wavelength is located middle of the two adjacent lasing modes. However, the difference decreases (worst case performance improves), when we decrease the front facet reflectivity of the F-P LD. There are two major improvements with the anti-reflection coating. One is the increase of the injection efficiency due to reduction of reflection at the front facet. The other is improvement of minimum fiber-to-fiber gain of the injected signal. This feature can be originated by the increase of the lasing threshold gain. It may be noted that the high reflection coating on the back facet improves the injection gain of the ASE signal.

Besides the front facet reflectivity, the cavity length of the laser is one of the major parameters, since the 


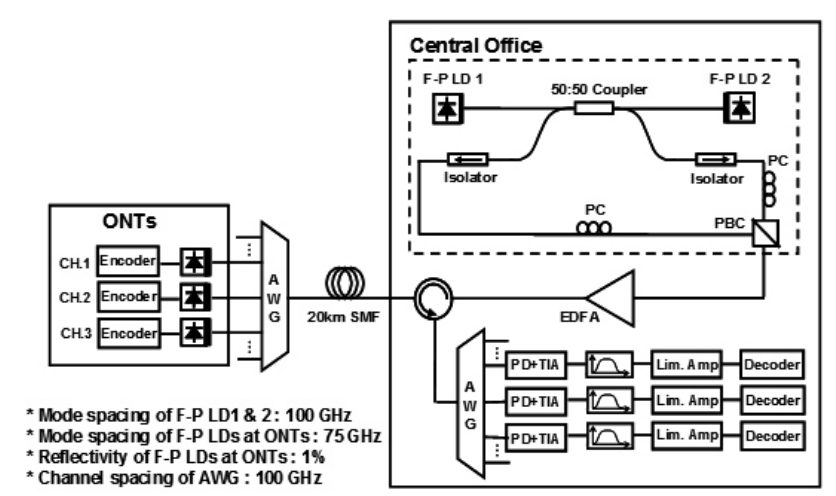

FIG. 5. Experimental setup for WDM-PON with proposed BLS.

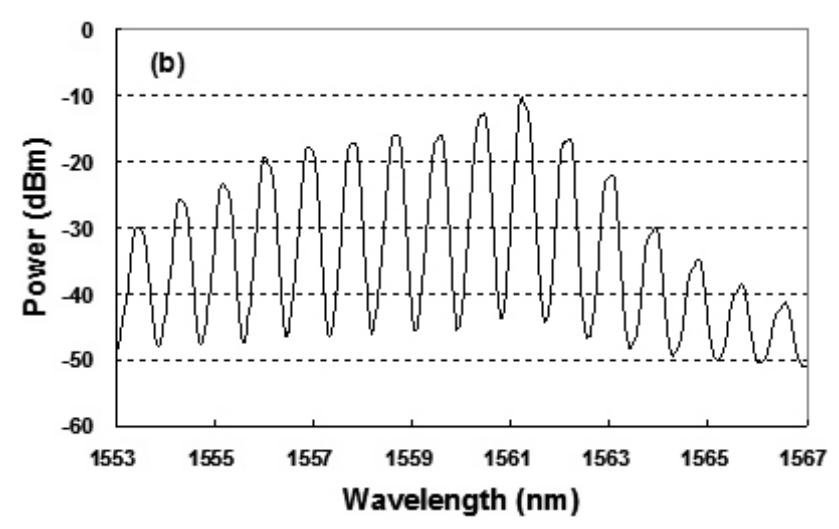

FIG. 6. The measured optical spectra of the proposed BLS output.

injection bandwidth should be comparable to the resonance frequency difference defined by the cavity length. In this paper we assume that the cavity length is 600 $\mu \mathrm{m}$. This is determined by the practical consideration of many parameters, such as channel spacing and packing limit.

For $100 \mathrm{Mb} / \mathrm{s}$ signal transmission at $100 \mathrm{GHz}$ channel spacing, $1 \%$ front facet reflectivity is good for color-free operation. However, we need to reduce the front facet reflectivity up to $0.1 \%$ for $1 \mathrm{~Gb} / \mathrm{s}$ signal transmission. We also need to increase the injection power for higher bit rate transmission. With increase of the injection power, the difference between the resonant case and the non-resonant case decreases due to saturation of the gain. Higher injection power also helps to reduce RIN of the output power. It may be noted that RSOA can be considered as an F-P LD with a very small front facet reflectivity. In this case, there is no difference between the resonant case and the non-resonant case. However, we need to take care of the temperature dependency.

The temperature dependence of the WDM-PON performance is one of the major engineering concerns. When temperature increases, many effects degrade the performance of the system. The material gain decreases as we increase the temperature. This effect can be overcome by increasing driving current into the laser. However, it increases the operating temperature of the laser due to self heating. Thus there exists a limit on the injection current. The limit depends on the thermal capacity of the laser package. If the thermal resistance is not small enough, thermal runaway exists. Another critical effect is increase of the saturation power of the F-P LD. Since F-P LD itself is an optical amplifier, the gain saturates as we increase the input power. Then, the output intensity noise is suppressed. Fig. 2 (d) show how the intensity noise is suppressed, when we operate the F-P LD or RSOA in a saturation region. The saturation power is $1 /\left(G_{t}\right)$, where $G$ is differential gain and $t_{s}$ is the effective lifetime of the carrier at the operating point. When we increase temperature, the Auger recombination rate increases [13]. Actually, the Auger recombination rate is an exponential function of the temperature. So, the saturation power increases although we increase the injection current to recover the gain. As a result, the F-P LD or RSOA is pushed to the linear region. Thus intensity noise suppression due to gain saturation diminishes. These features limit high temperature operation of the F-P LD or RSOA. Thus, amplifier with high thermal resistance or with low efficiency requires cooling at high temperature. The RSOA needs a tilted stripe or windowed facet to reduce front facet reflectivity to a certain value. It increases the internal loss of the gain medium. As a result, it may require cooling for high temperature operation.

\section{B. Low noise BLS}

The output of the wavelength locked F-P LD has high noise compared to a coherent DFB laser output, since we are injecting incoherent broadband light. This feature is not desirable for narrow spacing WDM-PON and/or high speed WDM-PON. The low noise BLS is desirable for these applications. The low noise BLS can be implemented as shown in the dotted box of Fig. 5. [14]. It consists of two AR-coated F-P LDs, a 50:50 optical coupler, and two isolators. The output of F-P LD 1 is injected to the F-P LD 2, and vice versa. We realized an unpolarized BLS by polarization multiplexing with two polarization controllers (PCs) and a polarization beam combiner (PBC). The measured 3-dB linewidth of a single mode of solitary F-P LD was less than 0.06 $\mathrm{nm}$. After mutual injection, the linewidth broadens up to $0.2 \mathrm{~nm}$ as shown in Fig. 6. The output spectrum of the proposed BLS looks like the spectrum sliced broadband light.

To investigate the feasibility of the proposed light source as a BLS, we measured the RIN of a filtered single mode among multiple modes of the mutually injected laser. We measured a similar RIN characteristic for each mode of the mutually injected laser output whose power is within $10 \mathrm{~dB}$ from the peak power. The 


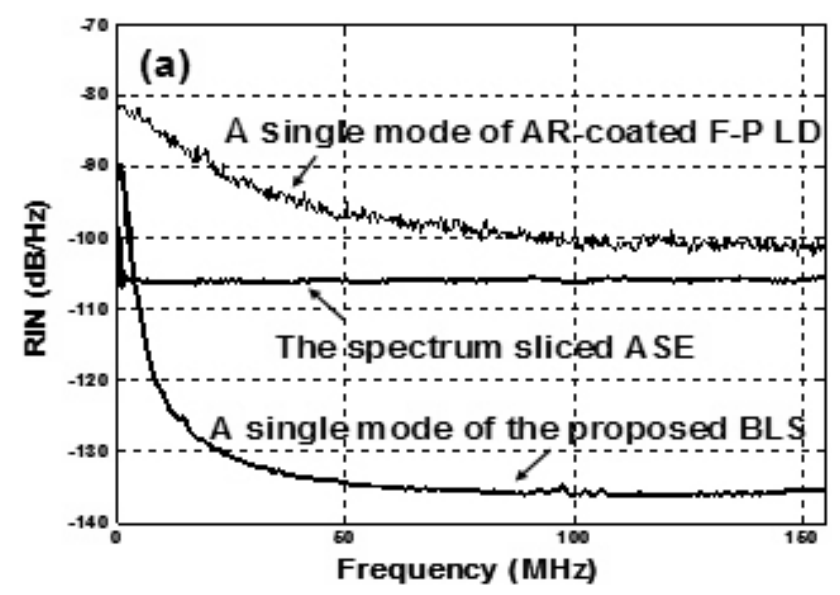

FIG. 7. The measured RIN of a single mode of the proposed BLS, a single mode of AR-coated F-P LD 1 and the spectrum sliced ASE.

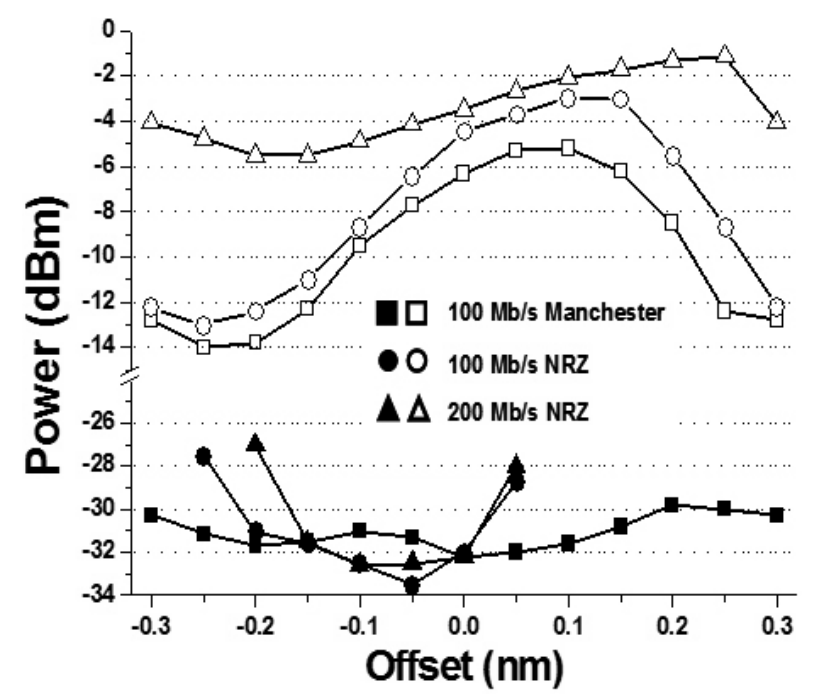

FIG. 8. The sensitivity at BER of $10^{-10}$ (solid markers) and the signal power of wavelength locked F-P LD (hollow markers) as a function of offset.

filtered mode of the proposed BLS shows the considerably suppressed RIN characteristic compared with a single mode of the solitary AR-coated F-P LD as shown in Fig. 7. The RIN was also compared with that of a spectrum sliced ASE from EDFA based BLS. The spectrum sliced ASE has the spectral width of $0.2 \mathrm{~nm}$. The achieved RIN by the mutual injection is considerably lower than the RIN of the spectrum sliced ASE. However, the RIN spectrum of the proposed BLS shows $1 / \mathrm{f}$ noise within a few $\mathrm{MHz}$ range as shown in Fig. 7 . Thus it is better to use Manchester coded data as a modulation format.

The realized low noise BLS was replaced with the BLS based on EDFA. The BLS output was amplified by an EDFA and transmitted through 20-km SMF. The F-P LD was modulated with NRZ data or Manchester coded data. For the latter case, we used a band-pass filter $(\mathrm{BPF})$ at the receiver to remove the $1 / \mathrm{f}$ noise in the transmitted signal. The low frequency cut-off of the BPF was optimized at $0.8 \mathrm{MHz}$. For both modulation formats, we maintained the signal data rate at 100 $\mathrm{Mb} / \mathrm{s}$, i.e., the transmitted bit rate of Manchester coded data is equivalent to $200-\mathrm{Mb} / \mathrm{s}$ NRZ data. To investigate the effects of Manchester coding, we compared the BER performance of Manchester coded data with that of NRZ data. As shown in Fig. 8, we examined the sensitivity at a BER of $10^{-10}$ (solid markers) and the signal power of the wavelength locked F-P LD (hollow markers) as a function of the offset that is the difference between injection wavelength and peak wavelength of a LD mode. The injection powers (total power) for $100-\mathrm{Mb} / \mathrm{s}$ Manchester, 100-Mb/s NRZ and 200-Mb/s NRZ were $-13 \mathrm{dBm},-11 \mathrm{dBm}$, and $-4 \mathrm{dBm}$, respectively.

For Manchester coded data, we measured the sensitivity difference of $2 \mathrm{~dB}$ within a whole offset range of $0.6 \mathrm{~nm}$ that is the mode spacing of the F-P LD at an ONT. We do not observe any sign of error-floor. The minimum link budget that is the signal power minus the receiver sensitivity was $17 \mathrm{~dB}$. However, BER of $10^{-10}$ was observed within a limited range of offset for NRZ data even though we increased the injection power by $3 \mathrm{~dB}$. Thus, we only achieved the color-free operation with Manchester coded data.

We decreased the cavity length to increase the transmission data rate. The $622 \mathrm{Mb} / \mathrm{s}$ data was transmitted with a low noise BLS with external cavity length of 12 $\mathrm{cm}$. The quality of the BLS can be increased by introducing a radio frequency $(\mathrm{RF})$ modulation signal into an F-P LD. We increase the 3-dB linewidth, while decrease the RIN by detuning the modulation frequency from the fundamental beat frequency determined by the passive cavity [33].

\section{RECENT RESEARCH AND DEVELOPMENT}

In an effort to increase the capacity of the WDM-PON, we demonstrate a WDM-PON with $50 \mathrm{GHz}$ channel spacing [16]. The number of subscribers in a single PON can be further increased by using hybrid approaches, i.e., a single wavelength can be shared by employing TDMA [17] or sub-carrier multiple access (SCMA) [18]. A hybrid WDM/TDM-PON that can serve 128 subscribers at data rates of $1.25 \mathrm{~Gb} / \mathrm{s}$ downstream and 622 $\mathrm{Mb} / \mathrm{s}$ upstream was demonstrated by cascading $1 \times 16$ AWG and $1 \times 8$ splitters [15]. Each of 16 100-GHz-spaced WDM channels was shared by 8 subscribers in the time domain. However, this requires a media access controller and ranging, as in a conventional TDM-PON. Another approach was proposed to maintain dedicated point-topoint connectivity. To do this, a wavelength was shared 


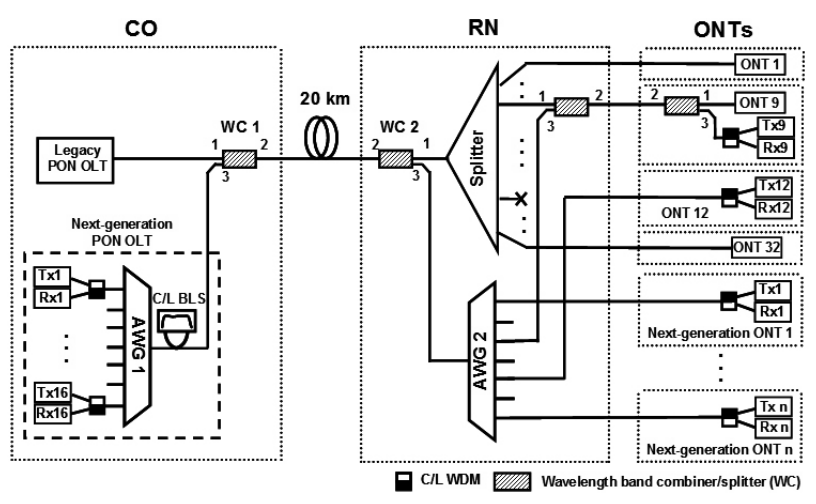

FIG. 9. The proposed evolution architecture from the TDM-PON to the next-generation PON.

by using SCMA in the RF domain [18]. The optical beat interference noise among multiple subscribers in a WDM channel was mitigated via forward error correction (FEC). When the channel spacing is $100 \mathrm{GHz}$, the total users in a single PON can be increased up to 192 with broadband BLSs. The color-free operation of ONTs was reported in both cases.

In an effort to consolidate a metro network and an access network for simplification of the overall network and reduction of CAPEX and OPEX, a long-reach DWDM-PON was demonstrated [19]. A channel spacing of $50 \mathrm{GHz}$ was used to accommodate more users in a single PON. The lasing wavelength of the F-P LD was matched to the injection wavelength of the spectrumsliced BLS in order to have a higher link budget and to reduce the required injection power. While transmitting 100 Base-x Ethernet packets (data rate $=125 \mathrm{Mb} / \mathrm{s}$ ) as the upstream and the downstream data, $70 \mathrm{~km}$ transmission without an optical amplifier was demonstrated [19].

It may be noted that the bit rate transparency of the WDM-PON can be used to support lifeline communication for an emergency voice communication channel. The transceiver can be operated at a voice bit rate to reduce the power consumption. A conventional coin type battery can be used for the lifeline communication [20].

Since a WDM-PON uses a wavelength selective device in a transmission path, e.g., AWG, it is not well suited for the delivery of broadcasting services. However, there have been some efforts to solve this problem. The delivery of broadcast video signals by using the cyclic property of the AWG was demonstrated [21]. The downstream data were transmitted with multi-channel DFB LDs at the $1550 \mathrm{~nm}$ band, while the upstream data were transmitted with light emitting diodes (LEDs) at the $1310 \mathrm{~nm}$ band. A DFB-LD operating at $1530 \mathrm{~nm}$ was used for broadcasting the signals of more than 70 digital video channels. To use a single DFB LD for the broadcast signal, an optical power splitter and an $\mathrm{N} x \mathrm{~N}$ cyclic
AWG were used. In a different approach, a sub-carrier multiplexed QAM data and base band digital signal were combined to generate a downstream signal for the subscriber. Then, a DFB LD was used for transmission of the downstream signal. At the ONT, a baseband receiver and an analog receiver were used and the re-modulation scheme with a RSOA was used for the upstream data transmission [22]. A low-noise BLS can be used to generate a multiple wavelength broadcast signal with an external modulator. The periodic property of the AWG can then be used to transmit the broadcast signal at an unused band [23].

For IP video services that require large bandwidth and high QoS, service availability is one of the critical concerns for the service provider. To increase service availability, protection from fiber faults and a fault localization method to reduce the recovery time are necessary. The wavelength-locked F-P LD has been used as a tunable optical time domain reflectometry (OTDR) with a tunable filter instead of a tunable laser [24]. A low power BLS of $-3 \mathrm{dBm}$ (total power) and a thermally tunable filter can be used to realize a low cost OTDR [25]. Another fault-localization scheme entailing monitoring of the status of the upstream signals was proposed [26]. When a failure is detected at an upstream channel, the corresponding downstream light source is switched to transmit OTDR pulses instead of data.

A smooth evolution scheme from the installed TDMPON to WDM-PON was also proposed and demonstrated [27] as shown in Fig. 9. The TDM-PON can be Broadband PON (B-PON), E-PON, or Gigabit PON (G-PON). The basic assumption for these TDM-PONs is that the upstream and the downstream wavelength bands follow the standard wavelength bands defined by ITU-T. The upstream and the downstream wavelength bands are $1,260 \mathrm{~nm} \sim 1,360 \mathrm{~nm}$ and $1,480 \mathrm{~nm} \sim 1,500 \mathrm{~nm}$, respectively. The WDM-PON and existing TDM-PON share feeder fiber by adding a three-port wavelength combiner/ splitter (WC) both at the OLT and the RN. The WDM-PON signal is combined with the TDM-PON signal by the WC at the OLT. They are then separated by a WC located at the RN before splitting. The optical power splitter is connected after the WC. Since the WDM signal does not go through the TDM-PON $\mathrm{ONU} / \mathrm{Ts}$, and vice versa, it is possible to design $\mathrm{ONU} / \mathrm{Ts}$ that are independent of each other. Furthermore, to deceases operation cost and provisioning time by a field reconfiguration of craft-man at the $\mathrm{RN}$, we propose a new remotely reconfigurable $\mathrm{RN}$ [28] to provide an efficient evolution path to NG services as shown in Fig. 10. A new remotely reconfigurable $\mathrm{RN}$ for next-generation services was proposed and demonstrated. Instantaneous optical powering to the $\mathrm{RN}$ with a photovoltaic converter and optical latch switches (OLSs) can reconfigure the optical path remotely and maintain the $\mathrm{RN}$ in a passive state. We believe that this can be easily achieved by 


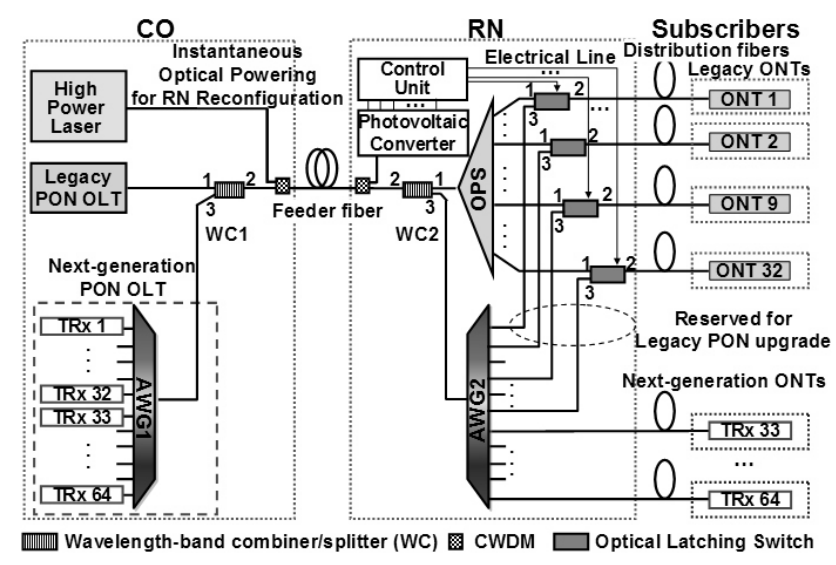

FIG. 10. Network architecture with a new remotely reconfigurable $\mathrm{RN}$ for the evolution to next-generation access networks.

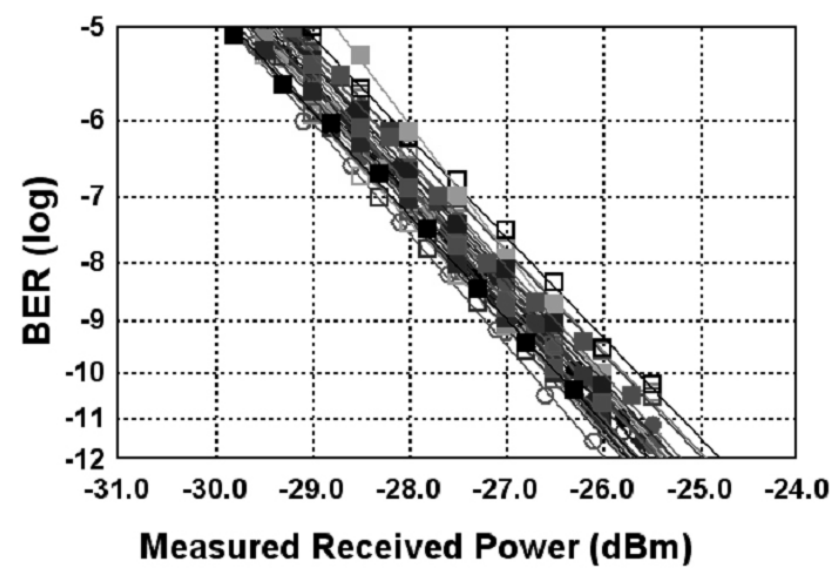

FIG. 11. Measured BER result of 32 channel after 15 $\mathrm{km}$ transmission for $\operatorname{ch} 1 \sim \operatorname{ch} 32$.

using a high power laser and a sufficiently wide dynamic range is provided. Thus, the proposed architecture can provide an efficient and cost-effective evolution path for next-generation services, while maintaining the $\mathrm{RN}$ in a passive state.

As a low cost approach, we proposed WDM-PON using double contact F-P LD without a seed light injection [29]. The un-cooled double-contact F-P LD is used to compensate the lasing envelope shift with temperature variation. Using this property, we can achieve a sufficient power margin to transmit $155-\mathrm{Mb} / \mathrm{s}$ data over 20-km SMF to make up for the filtering loss by AWGs. To avoid the high MPN, we use a spectral window that shows a low RIN. We used binary phase-shift keying (BPSK) as a modulation format to utilize a low RIN window. We demonstrated an error-free transmission with a 200-GHz channel spaced WDM-PON using the proposed method. It is also possible to realize a $100-$ $\mathrm{GHz}$ channel spaced WDM-PON using an optical amplifier or high power F-P LDs. As an another method, we propose spectrum-sliced Fabry-Pérot Laser Diodes
[30] by utilizing multiple peaks (several longitudinal modes) by the cyclic characteristics of the AWG.. This method is a simple and also achieved color-free operation.

For a high capacity WDM-PON, KT formed a consortium to develop a WDM-PON with capacity of more than $20 \mathrm{~Gb} / \mathrm{s}(1.25 \mathrm{~Gb} / \mathrm{s}$ times 16$)$. Three different technologies, a wavelength locked F-P LD, re-modulation of downstream data, and a coherent seeding method, were investigated by this consortium. The first scheme uses F-P LDs at both OLT and ONT with BLSs, as described in Sections II. The last two schemes use RSOA at the ONT. The remodulation scheme uses DFB LDs at the OLT. The coherent seeding scheme uses RSOA or DFB LD for downstream data. However, an extra array of DFB LDs is required for seed light [31].

Recently, we demonstrated WDM-PON of $40 \mathrm{~Gb} / \mathrm{s}$ over $15 \mathrm{~km}$ SMF [32]. In this system, the transmission length was limited by the available injection power and receiver sensitivity. We measured BER curves for all 32 channels. All BER curves show an error free transmission as shown in Fig. 11. The sensitivities were ranged from $-24.8 \mathrm{dBm}$ to $-26.0 \mathrm{dBm}$ at the BER of $10^{-12}$ The sensitivity difference between channels is only less than $1 \mathrm{~dB}$. It implies that the signal quality of the transmitted data is fairly uniform under uncontrolled injection ASE power and injection position for each F-P LD. This feature implies a feasibility color-free operation of ONTs. In addition, the dispersion penalty over $30 \mathrm{~km}$ transmission was $0.5 \mathrm{~dB}$. Thus, we can extend the transmission length with a high power BLS. The demonstrated WDMPON also shows a robust transmission performance on the optical back reflection [34]. Moreover, the transmission length can be extended further by employing a forward error correction (FEC). It may be noted that FEC can be also used to reduce the injection ASE power. We believe that we can accommodate to expand up to $2.5 \mathrm{~Gb} / \mathrm{s}$ at each subscriber. To have higher bit rate per channel, we need higher injection power and/or less noise in the injected ASE source. A proper operation of the F-P LD and/or RSOA having the high noise suppression is also helpful for higher speed operation.

\section{SUMMARY}

We reviewed wavelength locking technology which has been used for commercially deployed WDM-PON. A multimode F-P LD output can be converted to a quasi single mode with injection of a spectrum sliced ASE or low noise BLS. Then, we can use this light source for high speed WDM signal transmission. The advantage of this source is a color-free (colorless) operation. We can use a single type F-P LD for every ONT. This feature solves many technical and practical issues in a WDM-PON. In addition, technical advance in the athermal AWG eliminates thermal drift of transmission 
wavelength of an AWG located at the field. These features have brought commercial WDM-PON systems which have been deployed in Korea, Europe, and US.

We are investigating many aspects of wavelength locking characteristics to improve transmission length, number of channels, and transmission speed of the WDMPON. We achieved $80 \mathrm{~km}$ reach with $50 \mathrm{GHz}$ spacing for long reach PON. We also demonstrate $40 \mathrm{~Gb} / \mathrm{s}$ capacity WDM-PON over $15 \mathrm{~km}$ transmission length. A broadcasting method was also studied to accommodate digital TV signals in the WDM-PON. To upgrade TDMPON installed prior to the WDM-PON, we proposed a few ideas for seamless evolution method from the TDM-PON to the WDM-PON. We also proposed a low noise BLS that enables WDM-PON with narrower channel spacing.

We believe that the performance of the WDM-PON will be enhanced for future broadband band applications. The wavelength locking method will be one of key technologies for WDM-PON.

\section{REFERENCES}

[1] K. Cho, K. Fukuda, H. Esaki, and A. Kato, "The impact and implication of the growth in residential user-to-user traffic," in Proc. ACM/SIGCOMM (SIGC OMM2006), Pisa, Italy, Sep. 11-15, pp. 207-218, 2006.

[2] C. Ollivry, "Why Fiber? Why Now?," FTTH Council Europe, Montpellier, 2004.

[3] C.-H. Lee, W. Sorin, and B.-Y. Kim, "Fiber to the home using a PON infrastructure," IEEE J. Lightwave Tech., vol. 24, no. 12, pp. 4568-4583, 2006.

[4] S. Hornung, D. Payne, and R. Davey, "New architecture for an all optical network." in Proceedings of the Optical Fiber Communication Conference, Anaheim, CA, OTuH7, 2007.

[5] U. Hilbk, M. Burmeister, B. Hoen, Th. Hermes, and J. Saniter, "Selective OTDR measurements at the central office of individual fiber links in a PON," in Proceedings of the Optical Fiber Communication Conference, TuK3, 1997.

[6] K. Okamoto, Fundamentals of Optical Waveguides, (Academic Press, 2006).

[7] L. A. Coldren, G. A. Fish, Y. Akulova, J. S. Barton, and C. W. Coldren, "Tunable semiconductor lasers: A tutorial," IEEE J. Lightwave Tech., vol. 22, no. 1, pp. 193-202, 2004.

[8] H. D. Kim, S.-G. Kang, and C.-H. Lee, “A low-cost WDM source with an ASE injected Fabry-Pérot semiconductor laser," IEEE Photon. Technol. Lett., vol. 12, no. 8, pp. 1067-1069, 2000.

[9] H. Healey, P. Townsend, C. Ford, L. Johnston, P. Townley, I. Lealman, L. Rivers, S. Perrin, and M. Moore, "Spectral slicing WDM-PON using wavelength-seeded reflective SOAs," Electron. Lett., vol. 37, no. 19, pp. 1181-1182, 2001.

[10] Chang-Hee Lee, Sang-Mook Lee, Ki-Man Choi,
Jung-Hyung Moon, Sil-Gu Mun, Ki-Tae Jeong, Jin-Hee Kim, and ByoungWhi Kim, "WDM-PON experiences in Korea," Journal of Optical Networking, vol. 6, no. 5, pp. 451-464, 2007.

[11] W. Hong, C.-K. Chan, L.-K. Chen, and F. Tong, “An optical network unit for WDM access networks with downstream DPSK and upstream remodulated OOK data using injection-locked FP laser," IEEE Photon. Technol. Lett., vol. 15, no. 10, pp. 1476-1478, 2003.

[12] Kun-Youl Park, Sil-Gu Mun, Ki-Man Choi, and Chang-Hee Lee, "A Theoretical Model of a WavelengthLocked Fabry-Pérot Laser Diode to the Externally Injected Narrow-Band ASE," IEEE Photon Technol. Lett., vol. 17, no. 9, pp. 1797-1799, 2005.

[13] Hongyun Meng and Chang-Hee Lee, "Temperature dependent saturation characteristics of wavelength locked Fabry-Perot laser diodes," International Conference on Advanced Information Technology, Shenzhen, China, July 28-31, 2008.

[14] Ki-man Choi, Jin-Serk Baik, and Chang-Hee Lee, "Broad-band Light Source Using Mutually Injected Fabry-Pérot Laser Diodes for WDM-PON," IEEE Photon. Technol. Lett., vol.17, no.12, pp. 2529-2531, 2005.

[15] D. J. Shin, D. K. Jung, J. K. Kee, Y. K. Oh, J. H. Lee, H. S. Kim, C. H. Lee, S. T. Hwang, J. H. Ko, Y. J. Oh, T. I. Kim, and C. S. Shim, "Transmission of HDTV and Ethernet data over a WDM-PON employing ASE-injected Fabry-Perot laser diodes," in Proceedings of the Optical Fiber Communication Conference 2004, WO3, 2004.

[16] S.-M. Lee, K.-M. Choi, S.-G. Mun, J.-H. Moon, and C.-H. Lee, "Dense WDM-PON based on wavelengthlocked Fabry-Perot laser diodes," IEEE Photon. Technol. Lett., vol. 17, no. 7, pp. 1579-1581, 2005.

[17] D. J. Shin, D. K. Jung, H. S. Shin, J. K. Kwon, S. T. Hwang, Y. J. Oh, and C. S. Shim, "Hybrid WDM/TDM-PON with wavelength-selection-free transmitters," IEEE J. Lightwave Tech., vol. 23, no. 1, pp. 187-195, 2005.

[18] J.-S. Baik and C.-H. Lee, "Hybrid WDM/SCMA-PON using wavelength-locked Fabry-Pérot laser diodes," IEEE Photon. Technol. Lett., vol. 18, no. 15, pp. 15851587, 2006.

[19] Sang-Mook Lee, Sil-Gu Mun, Min-Hwan Kim, and Chang-Hee Lee, "Demonstration of a Long-Reach DWDM-PON for Consolidation of Metro and Access Networks," IEEE J. Lightwave Tech., vol. 25, no.1, pp. 271-276, 2007.

[20] K. Hong, J.-S. Baik, and C.-H. Lee, "A method of lifeline communication in WDM passive optical networks," IEEE Photon. Technol. Lett., vol. 17, no. 5, pp. 1124-1126, 2005.

[21] E. S. Son, K. H. Han, J. K. Kim, and Y. C. Chung, "Bidirectional WDM passive optical network for simultaneous transmission of data and digital broadcast video service," IEEE J. Lightwave Tech., vol. 21, no. 8, pp. 1723-1727, 2003.

[22] T.-Y. Kim and S.-K. Han, "Reflective SOA-based bidirectional WDM-PON sharing optical source for up/downlink data and broadcasting transmission," 
IEEE Photon. Technol. Lett., vol. 18, no. 22, pp. 23502352, 2006.

[23] J.-H. Moon, K.-M. Choi, and C.-H. Lee, "Overlay of broadcasting signal in a WDM-PON,” in Proceedings of the Optical Fiber Communication Conference OFC 2006, OThK8, Mar., 2006.

[24] J.-H. Park, J.-S. Baik, and C.-H. Lee, "Fault-localization in WDM-PONs," Opt. Exp., vol. 15, no. 4, pp. 1461-1466, 2007.

[25] K. Tanaka, H. Izumita, N. Tomita, and Y. Inoue, "In-service individual line monitoring and a method for compensating for the temperature-dependent channel drift of a WDM-PON containing an AWGR using a 1.6 $\mu \mathrm{m}$ tunable OTDR,” Integrated Optics and Optical Fibre Communications, WE4A4, 1997.

[26] K. W. Lim, E. S. Son, K. H. Han, and Y. C. Chung, "Fault localization in WDM passive optical network by reusing downstream light sources," IEEE Photon. Technol. Lett., vol. 17, no. 12, pp. 2691-2693, 2005.

[27] Ki-man Choi, Sang-Mook Lee, Min-Hwan Kim, and Chang-Hee Lee, "An Efficient Evolution Method From TDM-PON to Next-Generation PON,” IEEE Photon. Technol. Lett., vol. 19, no. 9, pp. 647-649, 2007.

[28] Jong Hoon Lee, Ki-Man Choi, and Chang-Hee Lee, “A Remotely Reconfigurable Remote Node for NextGeneration Access Networks," IEEE Photon. Technol. lett., vol. 20, no.11, pp. 915-917, 2008.

[29] Sil-Gu Mun, Jung-Hyung Moon, Ki-Man Choi,
Jin-Serk Baik, and Chang-Hee Lee, “A WDM-PON Employing Fabry-Pérot Laser Diodes Without a Seed Light Injection," IEEE Photon. Technol. Lett., vol. 19, no. 21, pp.1729-1731, 2007.

[30] Hoon-Keun Lee, Hyuek-Jae Lee, Chang-Hee Lee, “A Simple and Color-Free WDM-Passive Optical Network Using Spectrum-Sliced Fabry-Pérot Laser Diodes," IEEE Photon. Technol. Lett., vol. 20, no. 3, pp. 220-222, 2008.

[31] S. J. Park, G. Y. Kim, and T. S. Park, "WDM-PON system based on the laser light injected reflective semiconductor optical amplifier," Opt. Fiber Technol., vol. 12, pp. 162-169, 2006.

[32] Sil-Gu Mun, Jung-Hyung Moon, Hoon-Keun Lee, Jun-Young Kim, and Chang-Hee Lee, "A WDM-PON with a $40 \mathrm{~Gb} / \mathrm{s}(32 \times 1.25 \mathrm{~Gb} / \mathrm{s})$ capacity based on wavelength-locked Fabry-Perot laser diodes," Opt. Exp., vol. 16, no. 15, pp. 11361-11368, 2008.

[33] Ki-Man Choi and Chang-Hee Lee, "Low-Noise Broadband Light Source with a RF Modulation for a Large Capacity and High bit-rate WDM-PON," OptoElectronics and Optical Communications Conference, ThL-3, Sydney, Australia, Jul., 2008.

[34] Jung-Hyung Moon, Ki-Man Choi, Sil-Gu Mun, and Chang-Hee Lee, "Effects of Back-Reflection in WDM-PONs Based on Seed Light Injection," IEEE Photon. Technol. Lett., vol. 19, no. 24, pp. 2045-2047, 2007. 